\title{
GAPS BETWEEN NONZERO FOURIER COEFFICIENTS OF CUSP FORMS
}

\author{
SOUMYA DAS AND SATADAL GANGULY \\ (Communicated by Kathrin Bringmann)
}

\begin{abstract}
We prove that for any even integer $k \geq 12$, there are positive constants $c$ and $X_{0}$ that depend only on $k$ such that for all nonzero cusp forms $f$ of weight $k$ for the full modular group, any interval $\left(X, X+c X^{1 / 4}\right)$ with $X>X_{0}$ must contain an integer $n$ with the $n$-th Fourier coefficient of $f$ nonzero.
\end{abstract}

\section{INTRODUCTION}

Fourier coefficients of modular forms are interesting objects and various questions regarding their properties have been investigated by many researchers. In this article we study the question of Serre of bounding the length of a string of consecutive zero Fourier coefficients of cusp forms of integral weight. In [Se81, Serre defined, for any positive integer $n$,

$$
i_{f}(n):=\max \left\{i \mid a_{f}(n+j)=0, \quad 0 \leq j \leq i\right\},
$$

where $a_{f}(n)$ is the $n$-th Fourier coefficient (at the cusp at $\infty$ ) of a cusp form $f$ and proved that $i_{f}(n) \ll_{f} n$ if $f$ is not a linear combination of forms with complex multiplication (CM). In the same paper he asks whether it is the case that

$$
i_{f}(n) \ll_{f} n^{\delta},
$$

for some $\delta, 0<\delta<1$. The answer to this is certainly affirmative, as the above is known to hold for $\delta=3 / 5$ by the well-known Rankin-Selberg estimate. For details, see the introduction in [KRW07, which also discusses another approach to Serre's question. The question remains, nevertheless, of reducing the size of the exponent $\delta$, and several researchers contributed towards answering this question and its several modifications. See, for example, BO01, Al03, Al05, AZa05, [AZ08, and [KRW07, the last one, due to Kowalski, Robert, and Wu, containing the strongest general result. They use new bounds on certain exponential sums and distribution of $\mathfrak{B}$-free numbers to show that $i_{f}(n) \ll_{f} n^{7 / 17+\varepsilon}$ for any $\varepsilon>0$ for any cusp form $f$ which is not a linear combination of forms with complex multiplication.

In this article we improve upon the above result for all cusp forms for the full modular group $\mathrm{SL}_{2}(\mathbb{Z})$ by exploiting some congruence relations established by Hatada Hat79] for eigenvalues of Hecke operators acting on the space $\mathcal{S}_{k}$ of cusp forms of level one. Our main theorem is:

Received by the editors August 13, 2012 and, in revised form, December 19, 2012.

2010 Mathematics Subject Classification. Primary 11F30; Secondary 11F11, 11N25.

Key words and phrases. Fourier coefficients of cusp forms, sums of two squares. 
Theorem 1. Given any even positive integer $k \geq 12$, there is a positive constant $c$ that depends only on $k$ such that for all nonzero cusp forms $f$ in $\mathcal{S}_{k}, a_{f}(n) \neq 0$ for some integer $n \in\left(X, X+c X^{1 / 4}\right)$ for all $X \geq 1154$.

Theorem 1 has the following immediate corollary.

Corollary 1. For any nonzero cusp form $f \in \mathcal{S}_{k}$, we have that

$$
i_{f}(n) \ll_{k} n^{1 / 4} \text {. }
$$

A crucial ingredient in our work is a collection of congruences due to Hatada Hat79] that he established using the theory of Hecke operators acting on integral Eichler cohomology. A typical one says

$$
\lambda_{f}(p) \equiv p+1 \quad(\bmod 8),
$$

where $p \neq 2$ is any prime and $\lambda_{f}(p)$ is the eigenvalue of the $p$-th Hecke operator acting on $\mathcal{S}_{k}$. From this congruence we first show (Proposition 1) that there is an integer $M_{f}$ such that if $n$ is a sum of two squares and $\left(n, M_{f}\right)=1$, then $\lambda_{f}(n) \neq 0$. A modification of the simple but efficient argument of Bambah and Chowla [BC47] shows the interval $(X, X+H)$ with $H \gg X^{1 / 4}$ contains at least one integer which is a sum of two squares (with the added coprimality condition) for all $X$ sufficiently large.

It is this coprimality condition that gives rise to the dependence of the implied constant on the weight $k$ in Theorem 1 The dependence is inherent in Lemma 1 that is needed to handle higher prime powers, and this dependence can be removed if we are able to handle the harder problem of counting the sequence of square-free integers $n$ that are sums of two squares, thus doing away with the need to appeal to Lemma 1. This problem has been related to estimation of the error term

$$
P(X):=\sum_{1 \leq n \leq X} r(n)-\pi(X)
$$

in the Gauss circle problem (see [IK04, Chap. 8]) by Zhai [Zha06] (see also Krat82]). A well-known bound on $P(X)$ gives the next theorem, in which the implied constant is absolute, but this comes at the cost of a larger exponent.

Theorem 2. For any fixed $\varepsilon>0$, there are positive constants $c$ and $X_{0}$ that depend only on $\varepsilon$ such that for any cusp form $f$ of level one, we have that $a_{f}(n) \neq 0$ for some $n \in\left(X, X+c X^{131 / 416+\varepsilon}\right)$ whenever $X>X_{0}$.

The Gauss circle problem is related to estimation of exponential sums, and a wellknown conjecture in the theory of exponential sums is the Exponent Pair Hypothesis (see [K04, Chap. 8], for example). Suppose $h$ is a smooth function on an interval $[N, 2 N]$ such that its derivatives satisfy the bound

$$
h^{(j)}(x) \asymp H N^{-j}
$$

for every $j \geq 0$, where $H \geq N$ is a constant that depends on $h$ and the implied constants depend only on $j$. Then the hypothesis says that

$$
\sum_{N<n \leq N^{\prime}} e^{2 \pi i h(n)} \ll N^{1 / 2} H^{\varepsilon}
$$

for any $\varepsilon>0$ and any $N^{\prime}$ with $N \leq N^{\prime} \leq 2 N$. We have the following conditional result. 
Theorem 3. Assume the Exponent Pair Hypothesis. For any fixed $\varepsilon>0$, there are positive constants $c$ and $X_{0}$ that depend only on $\varepsilon$ such that for any cusp form $f$ of level one, we have that $a_{f}(n) \neq 0$ for some $n \in\left(X, X+c X^{1 / 4+\varepsilon}\right)$ whenever $X>X_{0}$.

Remark 1. The reason we consider only the full modular group is due to the fact that the congruence of Hatada that we need is available only in this case. If similar congruence holds for a form of higher level, then this or some similar method should give the same result for such a form.

Remark 2. The idea of using congruences to prove existence of nonzero Fourier coefficients of modular forms in short intervals is not new. Indeed, Alkan AZ05. had previously observed that the Ramanujan congruences for the $\tau$-function could be used to show that $\tau\left(m^{2}+23 n^{2}\right) \neq 0$ for any integers $m$ and $n$, and using this he had obtained a short interval result similar to this for the Ramanujan $\Delta$-function.

Remark 3. Instead of estimating $i_{f}(n)$ for all $n$, one may also look for a very strong bound that is valid for almost all $n$ or for most $n$ in the sense of density. Several works on this type of question have been done by Alkan and Zaharescu. See Al03, Al05, AZa05, and AZ08. For example, it is proved in Al03 that $i_{f}(n) \ll_{f, \phi} \phi(n)$ for almost all $n$, where $\phi$ is essentially any function monotonically increasing to infinity. They have also considered the case where $n$ is restricted to vary over arithmetic progressions.

Remark 4. Bambah and Chowla [BC47] conjectured that there should be an integer expressible as sums of two squares in any interval of the form $\left(X, X+X^{\varepsilon}\right)$ for any $\varepsilon>0$ for all $X$ sufficiently large. It seems reasonable to conjecture that the same should hold under the extra assumption that the sum of two squares is coprime to an arbitrary but fixed integer $M$. If we assume this, then the proof of Theorem 1 shows that we should have the bound $i_{f}(n) \ll n^{\varepsilon}$ for nonzero cusp forms $f$ of level one.

In the rest of the paper we prove Theorem 1 in detail and sketch the proofs of Theorem 2 and Theorem 3.

\section{Notation AND PREPARATORY LEMmas}

For even integers $k \geq 12$ we denote the space of holomorphic cusp forms (with trivial nebentypus) of weight $k$ for the modular group $\mathrm{SL}_{2}(\mathbb{Z})$ by $\mathcal{S}_{k}$. We denote the $n$-th Fourier coefficent of a form $f$ in this space by $a_{f}(n)$; i.e.,

$$
f(z)=\sum_{n \geq 1} a_{f}(n) q^{n},
$$

where $q:=e^{2 \pi i z}$. If $f$ is a Hecke eigenform, we make the standard normalization

$$
a_{f}(1)=1,
$$

so that

$$
a_{f}(n)=\lambda_{f}(n),
$$

where $\lambda_{f}(n)$ denotes the eigenvalue of the $n$-th Hecke operator associated to $f$. For a Hecke eigenform $f$, let $K=K_{f}:=\mathbb{Q}\left(\left\{\lambda_{f}(n): n \geq 1\right\}\right)$ and let $O_{K}$ be its ring of integers. For $m \in \mathbb{Z}$ and $x, y \in O_{K}$, we denote $x-y \in m O_{k}$ by $x \equiv y(\bmod m)$. 
Now we state a lemma that combines the level one case of a lemma of Murty and Murty (see [MM07, Lemma 2.5]) and that of a lemma of Kowalski, Robert, and $\mathrm{Wu}$ (see [KRW07, Lemma 2.2]).

Lemma 1. Let $f \in \mathcal{S}_{k}$ be a Hecke eigenform. Then for all sufficiently large primes $p$, either $\lambda_{f}(p)=0$ or $\lambda_{f}\left(p^{n}\right) \neq 0$ for all $n \geq 1$. If all the Hecke eigenvalues $\lambda_{f}(n)$ are rational integers, then the above conclusion is true for all primes.

The next lemma summarises various congruences for Hecke eigenvalues at prime powers that follows from the work of Hatada Hat79. Only parts of it will be used in the proof, but we include the other cases for completeness.

Lemma 2. For any integer $n \geq 1$, we have the congruence relations

$$
\lambda_{f}\left(p^{n}\right) \equiv\left\{\begin{array}{llll}
(n+1) \quad(\bmod 4) & \text { if } p \equiv 1 & (\bmod 4), \\
1 & (\bmod 4) & \text { if } n \equiv 0 & (\bmod 2) \text { and } p \equiv-1 \quad(\bmod 4) \\
1 \quad(\bmod 3) & \text { if } n \equiv 0 & (\bmod 2) \text { and } p=2 .
\end{array}\right.
$$

Proof. Reducing the generating series

$$
\sum_{n \geq 0} \lambda_{f}\left(p^{n}\right) X^{n}=\frac{1}{1-\lambda_{f}(p) X+p^{k-1} X^{2}}
$$

modulo (4) and using the basic congruence (see [Hat79])

$$
\lambda_{f}(p) \equiv p+1 \quad(\bmod 4) \text { for } p \neq 2,
$$

we get

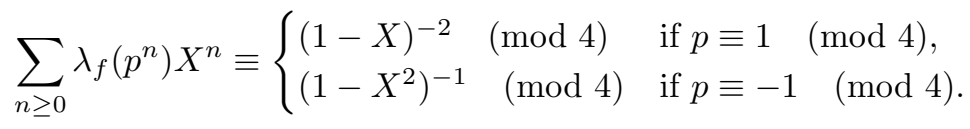

Using the negative binomial expansion

$$
(1-X)^{-t}=\sum_{s=0}^{\infty}\left(\begin{array}{c}
t+s-1 \\
s
\end{array}\right) X^{s}, \quad(t \geq 1),
$$

we finally get

$$
\lambda_{f}\left(p^{n}\right) \equiv\left\{\begin{array}{lll}
(n+1) \quad(\bmod 4) & \text { if } p \equiv 1 & (\bmod 4) \\
1 \quad(\bmod 4) & \text { if } n \equiv 0 & (\bmod 2) \text { and } p \equiv-1 \quad(\bmod 4) .
\end{array}\right.
$$

For the prime 2 we use another congruence from Hat79:

$$
\lambda_{f}(2) \equiv 0 \quad(\bmod 3) .
$$

We also note that $\lambda_{f}(2) \neq 0$ by a result of Kohnen Koh99]. Hence, reducing (2.1) modulo 3 , we obtain

$$
\lambda_{f}\left(2^{n}\right) \equiv 1 \quad(\bmod 3), \quad n \text { even }
$$




\section{Proofs of the theorems}

We begin this section with a crucial proposition which is an outcome of the above congruences.

Proposition 1. Let $f \in \mathcal{S}_{k}$ be a Hecke eigenform. Then there is an even integer $M_{f}$ such that

$$
\lambda_{f}\left(a^{2}+b^{2}\right) \neq 0
$$

for any integers $a$ and $b$, with $\left(a^{2}+b^{2}, M_{f}\right)=1$. If, moreover, all the Hecke eigenvalues $\lambda_{f}(n)$ are rational integers, then one can take $M_{f}=1$.

Proof. We consider the general case first. Let us write the prime factorization: $a^{2}+b^{2}=\prod_{j=1}^{s} p_{j}^{\alpha_{j}}$. Then, as is well known, either $p_{j}=2$ or the exponent $\alpha_{j}$ of $p_{j}$ is even if $p_{j} \equiv-1(\bmod 4)$. By multiplicativity of the Hecke eigenvalues $\lambda_{f}(n)$, we have

$$
\lambda_{f}\left(a^{2}+b^{2}\right)=\prod_{j=1}^{s} \lambda_{f}\left(p_{j}^{\alpha_{j}}\right) .
$$

Define $M_{f}$ to be the smallest even integer with the property that if $p$ is a prime and $\left(p, M_{f}\right)=1$, then $\lambda_{f}(p) \neq 0$ implies $\lambda_{f}\left(p^{n}\right) \neq 0$ for all $n \geq 1$. Existence of such integers is assured by Lemma 1. Let us call the set of primes coprime to $M_{f}$ to be $\mathcal{A}_{f}$. Let $p=p_{j} \in \mathcal{A}_{f}$. If $p \equiv 1(\bmod 4)$, then the congruence (2.2) implies that $\lambda_{f}(p) \neq 0$, whence it follows by Lemma 1 that $\lambda_{f}\left(p^{n}\right) \neq 0$ for all $n \geq 1$. If $p \equiv-1(\bmod 4)$, we invoke the second congruence relation in Lemma 2 to conclude $\lambda_{f}\left(p^{n}\right) \neq 0$ for all even $n$. Since $M_{f}$ is even, this finishes the proof.

If $\lambda_{f}(n) \in \mathbb{Z}$ for all $n \geq 1$, we make use of the last sentence in Lemma 1 and proceed as above. The congruences in Lemma 2 and the result of Kohnen that $\lambda_{f}(2) \neq 0$ ensure that none of the factors in the right-hand side of (3.1) can be zero.

Corollary 2. For any even integer $k \geq 12$, there is an integer $M_{k}$ such for all integers $a, b$ with $\left(a^{2}+b^{2}, M_{k}\right)=1$, the Hecke operator $T_{k}\left(a^{2}+b^{2}\right)$ is an automorphism of $\mathcal{S}_{k}$. One can take $M_{k}=1$ if the eigenvalues of all the Hecke operators are rational integers.

3.1. Proof of Theorem 1. Let us denote by $\mathcal{H}_{k}$ the normalized Hecke basis for $\mathcal{S}_{k}$ and define

$$
M:=\operatorname{lcm}\left\{M_{f} \mid f \in \mathcal{H}_{k}\right\} \quad\left(M_{f} \text { as in Proposition 1) }\right) .
$$

We note that $M$ depends only on $k$. We first prove Theorem 1 for a form $f \in \mathcal{H}_{k}$. Note that by Proposition 1, all we need to show is that there are integers prime to $M$ that can be written as sums of two squares in intervals of the type mentioned in the theorem. This is known in the case $M=1$ by the work of Bambah and Chowla [BC47]. We adapt their argument to treat the general case. We start with an integer $X$, sufficiently large, and choose an integer $m$ such that $m<X^{1 / 2}<m+1$. Define $\lambda$ to be the unique positive real that satisfies the equation $m^{2}+\lambda^{2}=X$. Let $m^{2} \equiv a(\bmod M)$, where $0 \leq a \leq M-1$. 
Claim. There exists an integer $b, 0 \leq b \leq M-1$, such that $\left(b^{2}+a, M\right)=1$. Granting the claim for a moment, we next choose an integer $\alpha$ such that $\alpha-1 \leq$ $(\lambda-b) / M<\alpha$. Let us define $n:=M \alpha+b(0 \leq b \leq M-1)$ with $b$ chosen as above. With these choices, note that $n-M \leq \lambda<n$. Thus we arrive at the inequalities

$$
m^{2}+n^{2}>m^{2}+\lambda^{2}=X, \quad m^{2}+n^{2} \leq m^{2}+(\lambda+M)^{2} \leq X+2 M \lambda+M^{2} .
$$

As in [BC47], one has $\lambda<3 X^{1 / 4}$ if $X \geq 1154$. This shows that

$$
m^{2}+n^{2}<X+6 M X^{1 / 4}+M^{2}<X+c X^{1 / 4},
$$

where $c>0$ is a constant that depends only on $M$ and thus only on the weight $k$.

It remains to prove the claim. Let us define the polynomial $A(x)=x^{2}+a$. We want to prove that $(A(b), M)=1$ for some $b$. Let the prime decomposition of $M$ be

$$
M=\prod_{i=1}^{g} q_{i}^{\beta_{i}} .
$$

It is enough to prove that there exists $b$ such that $\left(A(b), q_{i}\right)=1$ for all $1 \leq i \leq g$. Now we find integers $b_{i}$ such that $A\left(b_{i}\right) \not \equiv 0\left(\bmod q_{i}\right)$ for all $i$. This can be done easily. If $q_{i} \mid a$, we choose $b_{i}=q_{i}+1$; otherwise we choose $b_{i}=0$. Now, by the Chinese Remainder Theorem, we can choose an integer $b \equiv b_{i}\left(\bmod q_{i}\right)$ for all $i$. This finishes the proof as $A(b) \equiv A\left(b_{i}\right) \not \equiv 0\left(\bmod q_{i}\right)$ for all $i$.

If $f \in \mathcal{S}_{k}$ is a normalized Hecke eigenform with all its coefficients in $\mathbb{Z}$, then the above proof is much simplified as one can take $M=1$, and we record this special case as a proposition.

Proposition 2. Let $f \in \mathcal{S}_{k}$ be a normalized Hecke eigenform that has all its coefficients rational integers. Then, $\lambda_{f}(n) \neq 0$ for some integer $n \in\left(X, X+7 X^{1 / 4}\right)$ for all $X \geq 1154$.

Now we prove Theorem 1 by extending the result to all nonzero cusp forms following a technique of Balog and Ono [BO01. Let $f=\sum_{n \geq 1} a_{f}(n) q^{n}$ be any nonzero cusp form in $\mathcal{S}_{k}$. We can expand it as

$$
f=\sum_{i=1}^{\mu} c_{i} f_{i},
$$

where $\mu=\operatorname{dim} \mathcal{S}_{k}$ and the set $\left\{f_{i} \mid 1 \leq i \leq \mu\right\}$ is the normalized Hecke basis for $\mathcal{S}_{k}$. Let us write the Fourier expansion of each $f_{i}$ as

$$
f_{i}=\sum_{n \geq 1} \lambda_{i}(n) q^{n}, \quad \lambda_{i}(1)=1 .
$$

Without loss of generality we assume that $c_{1} \neq 0$. By the multiplicity one principle, for each pair $(i, j)$ with $i \neq j$, there are infinitely many primes $p$ such that $\lambda_{i}(p) \neq$ $\lambda_{j}(p)$. Let us choose the smallest prime $p_{1}$ such that $\lambda_{1}\left(p_{1}\right) \neq \lambda_{2}\left(p_{1}\right)$ and consider

$$
g_{2}:=T_{p_{1}} f-\lambda_{2}\left(p_{1}\right) f .
$$


It is clear that the Hecke eigenform $f_{2}$ does not appear in the decomposition of $g_{2}$ in terms of the Hecke basis as in (3.4). Furthermore, we can easily write the Fourier coefficients of $g_{2}$ :

$$
a_{g_{2}}(n)=a_{f}\left(p_{1} n\right)+p_{1}^{k-1} a_{f}\left(n / p_{1}\right)-\lambda_{2}\left(p_{1}\right) a_{f}(n) .
$$

We continue in this way $\mu-1$ times, removing the forms $f_{2}, f_{3}, \cdots, f_{\mu}$ one by one, and end up with a nonzero cusp form $F$ which must be of the form

$$
F=c^{\prime} f_{1}, \quad c^{\prime} \neq 0 .
$$

We see by induction that the Fourier coefficients of $F$ can be expressed as

$$
a_{F}(n)=\sum_{j=1}^{s} \beta_{j} a_{f}\left(n / r_{j}\right),
$$

for some complex numbers $\beta_{j}$ and some real numbers $r_{j}>0$. In fact, $\beta_{j}$ 's are algebraic integers and $r_{j}$ 's are rational numbers, but we shall not need this fact. Define

$$
R:=\max \left\{1, r_{j} \mid 1 \leq j \leq \mu\right\} .
$$

We note that $R$ depends only on $k$. Since we have already proved the theorem for Hecke eigenforms, applying it to $f_{1}$ we see that there is a constant $c>0$ that depends only on $k$ such that for every $j$ and for any $X \geq 1154 / r_{j}$, there is some $n \in\left(r_{j} X, r_{j} X+c X^{1 / 4}\right)$ such that $a_{F}(n) \neq 0$. Therefore, there is some $j$ for which $a_{f}\left(n / r_{j}\right) \neq 0$. Defining $X_{0}:=1154 R$, we see that all intervals $\left(X, X+c X^{1 / 4}\right)$ with $X>X_{0}$ must contain an integer $m$ for which $a_{f}(m) \neq 0$. This proves the theorem.

3.2. Proofs of Theorems $\mathbf{2}$ and $\mathbf{3}$. Now consider the sequence of square-free integers which are sums of two squares. Such integers are products of distinct primes that are congruent to $1(\bmod 4)$ and possibly the prime 2 . Therefore, by the result that $\lambda_{f}(2) \neq 0$ which follows from a work of Kohnen [Koh99] and the congruence (2.2), it follows that the Hecke eigenvalues corresponding to these integers are nonzero. Since no higher exponent is present, there is no need to appeal to Lemma 1, and thus the coprimality condition we had earlier is gone. This is how we remove the dependence of the constants on the form $f$. Now Theorem 2 follows from the best result known towards the existence of sums of two squares in short intervals due to Zhai Zha06. His result says that all intervals of the form $\left(X, X+X^{131 / 416+\varepsilon}\right)$ for any $\varepsilon>0$ contain square-free integers which are sums of two squares, provided $X$ is large enough. Zhai actually proves this result for any interval of the form $\left(X, X+X^{\theta+\varepsilon}\right)$ with $X$ sufficiently large, where $\varepsilon$ is arbitrary and $\theta$ is any positive real number such that the error term

$$
P(X):=\sum_{1 \leq n \leq X} r(n)-\pi(X)
$$

in the Gauss circle problem satisfies

$$
P(X) \ll_{\varepsilon} X^{\theta+\varepsilon}
$$

for any $\varepsilon>0$. Zhai's result follows from the bound

$$
P(X) \ll_{\varepsilon} X^{131 / 416+\varepsilon}
$$


due to Huxley [Hux03]. Obtaining the smallest $\theta$ such that the bound (3.7) holds is a famous problem in number theory, and it is conjectured that $\theta=1 / 4$ is possible. The Exponent Pair Hypothesis implies this conjecture (see [IK04, Chap. 8]) and thus Theorem 3.

\section{ACKNOWLEDGEMENTS}

The authors thank the School of Mathematics, T.I.F.R., Mumbai, and the StatMath Unit, I.S.I., Kolkata, where this work was carried out, for providing an excellent working atmosphere. It is a pleasure to thank W. Kohnen, from whom the authors first learned of the result of K. Hatada, and the anonymous referee for valuable suggestions.

\section{REFERENCES}

[Al03] Emre Alkan, Nonvanishing of Fourier coefficients of modular forms, Proc. Amer. Math. Soc. 131 (2003), no. 6, 1673-1680 (electronic), DOI 10.1090/S0002-9939-02-06758-8. MR.1953571 (2003k:11068)

[Al05] Emre Alkan, On the sizes of gaps in the Fourier expansion of modular forms, Canad. J. Math. 57 (2005), no. 3, 449-470, DOI 10.4153/CJM-2005-019-7. MR2134398 (2006a:11056)

[AZ05] Emre Alkan and Alexandru Zaharescu, Nonvanishing of the Ramanujan tau function in short intervals, Int. J. Number Theory 1 (2005), no. 1, 45-51, DOI 10.1142/S1793042105000029. MR2172330 (2006e:11054)

[AZa05] Emre Alkan and Alexandru Zaharescu, Nonvanishing of Fourier coefficients of newforms in progressions, Acta Arith. 116 (2005), no. 1, 81-98, DOI 10.4064/aa116-1-7. MR2114906 (2005i:11062)

[AZ08] Emre Alkan and Alexandru Zaharescu, On the gaps in the Fourier expansion of cusp forms, Ramanujan J. 16 (2008), no. 1, 41-52, DOI 10.1007/s11139-007-9091-z. MR2407238 (2009c:11061)

[BO01] Antal Balog and Ken Ono, The Chebotarev density theorem in short intervals and some questions of Serre, J. Number Theory 91 (2001), no. 2, 356-371, DOI 10.1006/jnth.2001.2694. MR1876282(2003h:11145)

[BC47] R. P. Bambah and S. Chowla, On numbers which can be expressed as a sum of two squares, Proc. Nat. Inst. Sci. India 13 (1947), 101-103. MR0022879(9,273a)

[Hat79] Kazuyuki Hatada, Eigenvalues of Hecke operators on SL(2, Z), Math. Ann. 239 (1979), no. 1, 75-96, DOI 10.1007/BF01420494. MR516060 (80b:10037)

[Hux03] M. N. Huxley, Exponential sums and lattice points. III, Proc. London Math. Soc. (3) 87 (2003), no. 3, 591-609, DOI 10.1112/S0024611503014485. MR2005876 (2004m:11127)

[IK04] Henryk Iwaniec and Emmanuel Kowalski, Analytic number theory, American Mathematical Society Colloquium Publications, vol. 53, American Mathematical Society, Providence, RI, 2004. MR2061214 (2005h:11005)

[Koh99] Winfried Hohnen, Period polynomials and congruences for Hecke algebras [eigenvalues], Proc. Edinburgh Math. Soc. (2) 42 (1999), no. 2, 217-224, DOI 10.1017/S0013091500020204. MR 1697394(2000c:11066)

[KRW07] Emmanuel Kowalski, Olivier Robert, and Jie Wu, Small gaps in coefficients of Lfunctions and $\mathfrak{B}$-free numbers in short intervals, Rev. Mat. Iberoam. 23 (2007), no. 1, 281-326, DOI 10.4171/RMI/496. MR2351136 (2008m:11100)

[Krat82] Ekkehard Krätzel, Squarefree numbers as sums of two squares, Arch. Math. (Basel) 39 (1982), no. 1, 28-31, DOI 10.1007/BF01899241. MR674530(84e:10057)

[Leh47] D. H. Lehmer, The vanishing of Ramanujan's function $\tau(n)$, Duke Math. J. 14 (1947), 429-433. MR.0021027 (9,12b)

[MM07] M. Ram Murty and V. Kumar Murty, Odd values of Fourier coefficients of certain modular forms, Int. J. Number Theory 3 (2007), no. 3, 455-470, DOI 10.1142/S1793042107001036. MR2352830 (2008i:11063) 
[Se81] Jean-Pierre Serre, Quelques applications du théorème de densité de Chebotarev (French), Inst. Hautes Études Sci. Publ. Math. 54 (1981), 323-401. MR644559 (83k:12011)

[Zha06] Wenguang Zhai, Square-free integers as sums of two squares, Number theory, Dev. Math., vol. 15, Springer, New York, 2006, pp. 219-227, DOI 10.1007/0-387-30829-6_15. $\operatorname{MR} 2213838(2006 \mathrm{k}: 11187)$

School of Mathematics, Tata Institute of Fundamental Research, Homi Bhabha RoAd, Mumbai - 400005, India

E-mail address: somu@math.tifr.res.in

E-mail address: soumya.u2k@gmail.com

Indian Statistical Institute, Theoretical Statistics and Mathematics Unit, 203 BarRaCkPore Trunk RoAd, Kolkata 700108, India

E-mail address: sgisical@gmail.com 